\title{
Nuclear Forces from Lattice QCD
}

Tetsuo Hatsuda* (for HAL QCD Collaboration) ${ }^{\dagger}$

Phys. Dep., Univ. of Tokyo, Tokyo 113-0033, Japan

E-mail: hatsuda@phys.s.u-tokyo.ac.jp

A method to extract nucleon-nucleon (NN) potentials from the Bethe-Salpeter amplitude in lattice QCD is presented. It is applied to the two nucleons on the lattice with quenched QCD simulations. By disentangling the mixing between the S-state and the D-state, we obtain central and tensor potentials in the leading order of the velocity expansion of the non-local NN potential. The spatial structure, the quark mass dependence and the velocity dependence of the NN potential are analyzed. Preliminary result in (2+1)-flavor QCD simulations is also shown.

6th International Workshop on Chiral Dynamics, CD09

July 6-10, 2009

Bern, Switzerland

\footnotetext{
${ }^{*}$ Speaker.

$\dagger$ This research was supported in part by the Grant-in-Aid for Scientific Research on Innovative Areas (No. 2004: 20105003).
} 


\section{Introduction}

The origin of the nuclear force is one of the major unsolved problems in particle and nuclear physics. To describe the elastic nucleon-nucleon (NN) scattering at low-energies and the deuteron properties, the notion of the NN potential turns out to be very useful [1]. The phenomenological NN potentials which can fit the NN data precisely are known to have the following properties: (i) The long range part (the relative distance $r>2 \mathrm{fm}$ ) is dominated by the one-pion exchange introduced by Yukawa [2]. (ii) The medium range part (1 fm $<r<2 \mathrm{fm}$ ) receives significant contributions from the exchange of two-pions $(\pi \pi)$ and heavy mesons $(\rho, \omega$, and $\sigma)$. (iii) The short range part $(r<1 \mathrm{fm})$ is best described by a strong repulsive core as introduced by Jastrow [3]. (iv) A strong attractive spin-orbit force in the isospin 1 channel exists at medium and short distances. (i) is related to the tensor force which is a key for the deuteron binding, (ii) is important for the binding of nuclei with more than 2 nucleons, (iii) is important for the stability of nuclei and neutron stars, and (iv) is related to the ${ }^{3} \mathrm{P}_{2}$ neutron pairing which leads to the neutron superfluidity inside neutron stars [4].

A repulsive core surrounded by an attractive well as seen in the phenomenological nuclear force is a common feature of the "effective" potentials between composite particles. The LenardJones potential between neutral atoms or molecules is a well-known example in atomic physics. The potential between ${ }^{4} \mathrm{He}$ nuclei is a typical example in nuclear physics. The origin of the repulsive cores in these examples are known to be the Pauli exclusion among electrons or among nucleons. The same idea, however, is not applicable to the NN potential, because the quark has not only spin and flavor but also color which allows six quarks occupy the same state without violating the Pauli principle. To account for the repulsive core of the NN force, therefore, various different ideas have been proposed so far [5]: an exchange of the neutral $\omega$ meson as proposed by Nambu [6], exchanges of non-linear pion field, a combination of the Pauli principle with the one-gluonexchange between quarks and so on. Despite all these efforts, convincing account of the nuclear force has not yet been obtained.

\section{NN interactions from lattice QCD}

Under the situation mentioned above, it is highly desirable to study the NN interactions from the first principle lattice QCD simulations. A theoretical framework suitable for such purpose was first proposed by Lüscher [7]: For two hadrons in a finite $L^{3}$ box, an exact relation between the energy spectrum in the box and the elastic scattering phase shift was derived: If the range of the hadron interaction $R$ is sufficiently smaller than the size of the box $R<L / 2$, the behavior of the Bethe-Salpeter (BS) wave function $\psi(\vec{r})$ in the interval $R<|\vec{r}|<L / 2$ under the periodic boundary conditions has sufficient information to relate the phase shift and the two-particle spectrum. The Lüscher's method bypasses the difficulty to treat the real-time scattering process on the Euclidean lattice. Furthermore, it utilizes the finiteness of the lattice box effectively to extract the information of the on-shell scattering matrix and the phase shift. This approach has been applied to the NN scattering lengths in [8].

Recently, we have proposed a closely related but an alternative approach to the NN interactions from lattice QCD [9, 10]. The starting point is the same BS wave function $\psi(\vec{r})$ : Instead of 
looking at the wave function outside the range of the interaction, we consider the internal region $|\vec{r}|<R$ and define an energy-independent non-local potential $U\left(\vec{r}, \vec{r}^{\prime}\right)$ from $\psi(\vec{r})$ so that it obeys the Schrödinger type equation in a finite box. Since $U\left(\vec{r}, \vec{r}^{\prime}\right)$ for strong interaction is localized in its spatial coordinates due to confinement of quarks and gluons, the potential receives finite volume effect only weakly in a large box. Therefore, once $U$ is determined and is appropriately extrapolated to $L \rightarrow \infty$, one may simply use the Schrödinger equation in the infinite space to calculate the scattering phase shifts and bound state spectra to compare with experimental data. Further advantage of utilizing the potential is that it would be a smooth function of the quark masses so that it is relatively easy to handle. This is in sharp contrast to the the scattering length which shows a singular behavior around the quark mass corresponding to the formation of the $\mathrm{NN}$ bound state [11].

Since we consider the non-asymptotic region $(|\vec{r}|<R)$ of the wave function, the resultant potential $U$ and the $T$-matrix are off-shell. Therefore, they depend on the nucleon interpolating operator adopted to define the BS wave function. This is in a sense an advantage, since one can establish a one-to-one correspondence between the nucleon interpolating operator and the NN potential in $\mathrm{QCD}$, which is not attainable in phenomenological NN potentials. It also implies that the NN potential on the lattice and the phenomenological NN potentials are equivalent only in the sense that they give the same phase shifts, so that the comparison of their spatial structures should be made only qualitatively.

\section{Non-local potential from the BS wave function}

Let us consider the following BS wave function for the 6-quark state with total energy $W$ and the total three-momentum $\vec{P}=\overrightarrow{0}$ in a finite box; $\Psi_{\alpha \beta}(\vec{r}=\vec{x}-\vec{y}, t)=\left\langle\operatorname{vac}\left|n_{\beta}(\vec{y}, t) p_{\alpha}(\vec{x}, t)\right| W, \vec{P}=\right.$ $\overrightarrow{0}\rangle \equiv \psi_{\alpha \beta}(\vec{r}) e^{-i W t}$. The local composite operators for the proton and the neutron are denoted by $p_{\alpha}(\vec{x}, t)$ and $n_{\beta}(\vec{y}, t)$ with spinor indices $\alpha$ and $\beta$. The state $|W, \vec{P}=\overrightarrow{0}\rangle$ is a QCD eigenstate with baryon number 2 and with the same quantum numbers as the pn system. One should keep in mind that $|W, \vec{P}=\overrightarrow{0}\rangle$ is not a simple superposition of a product state $|\mathrm{p}\rangle \otimes|\mathrm{n}\rangle$, since there are complicated exchanges of quarks and gluons between the two composite particles.

The spatial extent of the NN interaction in QCD is short ranged and is exponentially suppressed beyond the distance $R>2 \mathrm{fm}$. Therefore, the spatial part of the BS wave function in the "outer region" $(r>R)$ satisfies the Helmholtz equation below the pion production threshold, $\left(\nabla^{2}+k^{2}\right) \psi_{\alpha \beta}(\vec{r})=0$, up to an exponentially small correction. Then we can define the non-local potential $U$ from $\psi$ and $k^{2}$ measured on the lattice:

$$
\begin{aligned}
\left(E-H_{0}\right) \psi_{\alpha \beta, E}(\vec{r}) & =\int U_{\alpha \beta ; \gamma \delta}\left(\vec{r}, \vec{r}^{\prime}\right) \psi_{\gamma \delta, E}\left(\vec{r}^{\prime}\right) d^{3} r^{\prime} \\
U_{\alpha \beta ; \gamma \delta}\left(\vec{r}, \vec{r}^{\prime}\right) & =V_{\alpha \beta ; \gamma \delta}(\vec{r}, \vec{v}) \delta\left(\vec{r}-\vec{r}^{\prime}\right)
\end{aligned}
$$

where $\vec{v}(=\vec{p} / \mu=-i \nabla / \mu)$ is the velocity operator. To make a formal resemblance with the non-relativistic case, we have introduced the "effective center of mass energy", $E=k^{2} /(2 \mu)=$ $k^{2} / m_{N}$ and the "free Hamiltonian", $H_{0}=-\nabla^{2} / m_{N}$. By construction, the solution of Eq.(3.1) with $U_{\alpha \beta ; \gamma \delta}\left(\vec{r}, \vec{r}^{\prime}\right)$ extrapolated to $L \rightarrow \infty$ reproduces the correct BS wave function in the asymptotic region, and hence the phase shifts and binding energies of the two-nucleon system. 
The simplest interpolating operators for the nucleon $N=(p, n)$ in terms of the quark field $q(x)$ would be $N_{\beta}(x)=\varepsilon_{a b c}\left(q_{a}(x) C \gamma_{5} q_{b}(x)\right) q_{c \beta}(x)$, with $a, b$ and $c$ being color indices and $C$ being the charge conjugation matrix. Such a local operator is most convenient for relating the BS wave function to the four-point Green's function and the scattering observables at $L \rightarrow \infty$. Closely related formulation was given long time ago by Nishijima, Zimmermann and Hagg who derived the generalized reduction formula for local composite fields [12].

In principle, one may choose any composite operators with the same quantum numbers as the nucleon to define the BS wave function. Different operators give different BS wave functions and different NN potentials, although they lead to the same observables. This is quite analogous to the situation in quantum mechanics where the unitary transformation of the wave function changes the structure of the potential while the observables are not modified. A theoretical advantage of our approach based on lattice QCD is that we can unambiguously trace the one-to-one correspondence between the NN potential and the interpolating operator in QCD as we mentioned.

The general form of the non-local NN potential $U$ or equivalently the velocity dependent NN potential $V$ in Eq.(3.2) in the two-component spinor space has been classified by Okubo and Marshak [13]. The leading order (LO) and the next-leading-oder (NLO) terms of the the velocity expansion of $V(\vec{r}, \vec{v})$ reads [14]

$$
\begin{aligned}
V= & V_{C}(r)+V_{T}(r) S_{12}+V_{L S}(r) L \cdot S+O\left(\vec{v}^{2}\right) \\
= & V_{0}(r)+V_{\sigma}(r)\left(\sigma_{1} \cdot \sigma_{2}\right)+V_{\tau}(r)\left(\tau_{1} \cdot \tau_{2}\right)+V_{\sigma \tau}(r)\left(\sigma_{1} \cdot \sigma_{2}\right)\left(\tau_{1} \cdot \tau_{2}\right) \\
& +\left[V_{T 0}(r)+V_{T \tau}(r)\left(\tau_{1} \cdot \tau_{2}\right)\right] S_{12} \\
& +\left[V_{L S 0}(r)+V_{L S \tau}(r)\left(\tau_{1} \cdot \tau_{2}\right)\right] L \cdot S+O\left(\vec{v}^{2}\right),
\end{aligned}
$$

where $V_{C}$ and $V_{T}$ are LO $\left(O\left(\vec{v}^{0}\right)\right)$ terms, while $V_{L S}$ is a NLO $(O(\vec{v}))$ term. On the lattice, it is relatively unambiguous to extract information for the orbital angular momentum states $\ell=0,1,2,3=$ S, P,D, F using the irreducible representations of the cubic group [7]. Then, at most 16 independent (14 diagonal and 2 off-diagonal) matrix elements of the potential are obtained, so that 8 unknown LO and NLO terms in Eq.(3.4) can be extracted in two different ways.

\section{Central and tensor forces from lattice QCD}

To define the BS wave function on the lattice, we start from the four-point correlator,

$$
\mathscr{G}_{\alpha \beta}=\left\langle\operatorname{vac}\left|n_{\beta}(\vec{y}, t) p_{\alpha}(\vec{x}, t) \overline{\mathscr{J}}_{p n}\left(t_{0} ; J^{P}\right)\right| \operatorname{vac}\right\rangle \rightarrow A_{0} \psi_{\alpha \beta}\left(\vec{r} ; J^{P}\right) \mathrm{e}^{-E_{0}\left(t-t_{0}\right)} \quad\left(t \gg t_{0}\right),
$$

where $A_{0}$ is an $r$-independent constant. The states created by the source $\overline{\mathscr{J}}_{p n}$ have the conserved quantum numbers, $\left(J, J_{z}\right)$ (total angular momentum and its z-component) and $P$ (parity). For studying the nuclear force in the $J^{P}=0^{+}\left({ }^{1} \mathrm{~S}_{0}\right)$ channel and the $J^{P}=1^{+}\left({ }^{3} \mathrm{~S}_{1}\right.$ and $\left.{ }^{3} \mathrm{D}_{1}\right)$ channel, we adopt a wall source with the Coulomb gauge fixing at $t=t_{0}$. The BS wave function in the orbital S-state is defined with the projection operator for the orbital angular momentum $\left(P^{(\ell)}\right)$ and that for the spin $\left(P^{(s)}\right)$ as $\psi\left(r ;{ }^{1} \mathrm{~S}_{0}\right)=P^{(\ell=0)} P^{(s=0)} \psi\left(\vec{r} ; 0^{+}\right)$and $\psi\left(r ;{ }^{3} \mathrm{~S}_{1}\right)=P^{(\ell=0)} P^{(s=1)} \psi\left(\vec{r} ; 1^{+}\right)$.

The asymptotic momentum $k$ for the S-states is obtained by fitting the BS wave function $\psi(\vec{r})$ with the Green's function $G\left(\vec{r} ; k^{2}\right)$ in a finite and periodic box satisfying $\left(\nabla^{2}+k^{2}\right) G\left(\vec{r} ; k^{2}\right)=$ 
$-\delta_{\text {lat }}(\vec{r})$ with $\delta_{\text {lat }}(\vec{r})$ being the periodic delta-function. The fits are performed outside the range of the NN interaction determined by $\nabla^{2} \psi(\vec{r}) / \psi(\vec{r})[15]$. The NN scattering lengths for the S-states can be deduced from the standard Lüscher's formula [7].

In the LO of the velocity expansion, only the central potential $V_{C}(r)$ and the tensor potential $V_{T}(r)$ are relevant: The central potential acts separately on the $\mathrm{S}$ and $\mathrm{D}$ components, while the tensor potential provides a coupling between these two. Therefore, we consider a coupled-channel Schrödinger equation in the $J^{P}=1^{+}$channel [16]:

$$
\left(H_{0}+V_{C}(r)+V_{T}(r) S_{12}\right) \psi\left(\vec{r} ; 1^{+}\right)=E \psi\left(\vec{r} ; 1^{+}\right) .
$$

Projections to the $\mathrm{S}$-wave and D-wave components are obtained as $\psi_{\alpha \beta}\left(r ;{ }^{3} \mathrm{~S}_{1}\right) \equiv P^{(\ell=0)} \psi_{\alpha \beta}\left(\vec{r} ; 1^{+}\right)$ and $\psi_{\alpha \beta}\left(r ;{ }^{3} \mathrm{D}_{1}\right) \equiv\left(1-P^{(\ell=0)}\right) \psi_{\alpha \beta}\left(\vec{r} ; 1^{+}\right)$. In the LO of the velocity expansion, it is sometimes useful to define the "effective" central potential $V_{C}^{\text {eff }}(r)[\overline{9}]: V_{C}^{\text {eff }}(r)=E+\frac{1}{m_{N}} \frac{\nabla^{2} \psi(r)}{\psi(r)}$. Note that $V_{\mathrm{C}}^{\text {eff }}(r)$ in the ${ }^{3} \mathrm{~S}_{1}$ channel contains the effect of $V_{T}(r)$ implicitely as higher order effects through the process such as ${ }^{3} \mathrm{~S}_{1} \rightarrow{ }^{3} \mathrm{D}_{1} \rightarrow{ }^{3} \mathrm{~S}_{1}$.

\section{Numerical results in quenched QCD}

In the quenched simulations, we employ the standard plaquette gauge action on a $32^{4}$ lattice with the bare QCD coupling constant $\beta=6 / g^{2}=5.7$. The corresponding lattice spacing is $1 / a=1.44(2) \mathrm{GeV}(a \simeq 0.137 \mathrm{fm})$ determined from the $\rho$ meson mass in the chiral limit. The physical size of our lattice then reads $L \simeq 4.4 \mathrm{fm}$. We adopt the standard Wilson quark action with the hopping parameter $\kappa=0.1640,0.1665,0.1678$, which correspond to $m_{\pi} \simeq 731,529,380$ $\mathrm{MeV}$, respectively. The periodic boundary condition is imposed on the quark fields along the spatial direction, while the Dirichlet boundary condition is imposed in the temporal direction at the time-slice $t=0$. The wall source is placed on the time-slice at $t_{0} / a \equiv 5$ with the Coulomb gauge fixing at $t=t_{0}$. The lowest effective c.m. energy $E$ in the above setup ranges from $-0.4 \mathrm{MeV}$ to $-1 \mathrm{MeV}$. Note that $E$ for scattering states can be negative in a finite box.

\subsection{Central and tensor forces in the ${ }^{3} S_{1}$ channel}

Shown in Fig. 1 is the central potential $V_{C}(r)$ and tensor potential $V_{T}(r)$ together with effective central potential $V_{C}^{\text {eff }}(r)$ in the ${ }^{3} \mathrm{~S}_{1}$ channel obtained in the LO velocity expansion. In the real world, $V_{\mathrm{C}}^{\text {eff }}(r)$ is expected to acquire sufficient attraction from the tensor force. This is the reason why bound deuteron exists in the ${ }^{3} \mathrm{~S}_{1}$ channel while the bound dineutron does not exist in the ${ }^{1} \mathrm{~S}_{0}$ channel. Now, we see from Fig. 1 that the difference between $V_{C}(r)$ and $V_{C}^{\text {eff }}(r)$ is still small in our quenched simulations due to relatively large quark masses.

The tensor potential $V_{T}(r)$ in Fig. 1 shows that it is negative for the whole range of $r$ with a minimum at short distance below $0.5 \mathrm{fm}$. If the long range part of the tensor force is dominated by the one-pion exchange as expected from the meson theory, $V_{T}(r)$ could be rather sensitive to the change of the quark mass. As shown in Fig. 2, it is indeed the case: Attraction of $V_{T}(r)$ is substantially enhanced as the quark mass decreases. For practical applications in nuclear physics, it is more useful to parametrize the lattice results by known functions. We have tried such a fit for $V_{T}(r)$ under the assumption of the one- $\rho$-exchange + one-pion-exchange with Gaussian form 


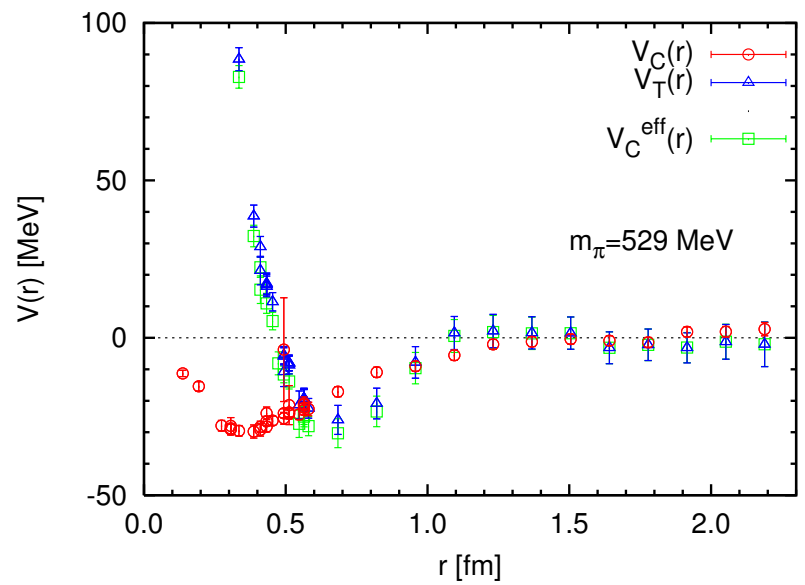

Figure 1: The central potential $V_{C}(r)$ and the tensor potential $V_{T}(r)$ obtained from the $J^{P}=1^{+}$BS wave function at $m_{\pi}=529 \mathrm{MeV}$.

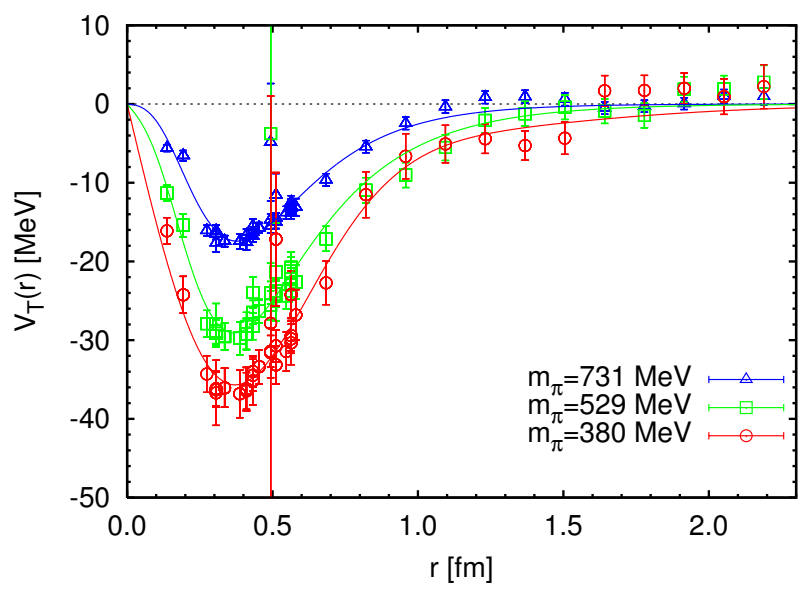

Figure 2: Quark mass dependence of tensor potential. The lines are the four-parameter fit using the one- $\rho$ exchange + one-pion-exchange with Gaussian form factors.

factors: $V_{T}(r)=b_{1}\left(1-e^{-b_{2} r^{2}}\right)^{2}\left(1+\frac{3}{m_{\rho} r}+\frac{3}{\left(m_{\rho} r\right)^{2}}\right) \frac{e^{-m_{\rho} r}}{r}+b_{3}\left(1-e^{-b_{4} r^{2}}\right)^{2}\left(1+\frac{3}{m_{\pi} r}+\frac{3}{\left(m_{\pi} r\right)^{2}}\right) \frac{e^{-m_{\pi} r}}{r}$. The results are shown by the solid lines in Fig. 2 .

\subsection{Velocity dependence of the potential}

So far we have considered the potential determined from the lattice data taken almost at zero effective c.m. energy $E \simeq 0 \mathrm{MeV}$. If the local potential determined from the other energies have different spatial structure, it is an indication of the velocity dependent terms. Such a velocity dependence has been recently studied by changing the spatial boundary condition of the quark field from the periodic one to the anti-periodic one [17]: On a $32^{3} \times 48$ lattice with the lattice spacing $a=0.137 \mathrm{fm}, 2000$ gauge configurations are accumulated. The minimum momentum is given by $\vec{p}_{\min }=(\pi, \pi, \pi) /(32 a)$, which leads to $\left|\vec{p}_{\min }\right| \simeq 240 \mathrm{MeV}$ and $E \simeq 50 \mathrm{MeV}$. In Fig.3, the central NN potential for the ${ }^{1} \mathrm{~S}_{0}$ state with $\mathrm{APBC}(E \simeq 50 \mathrm{MeV})$ is plotted as a function of $r$ at $t / a=9$, 


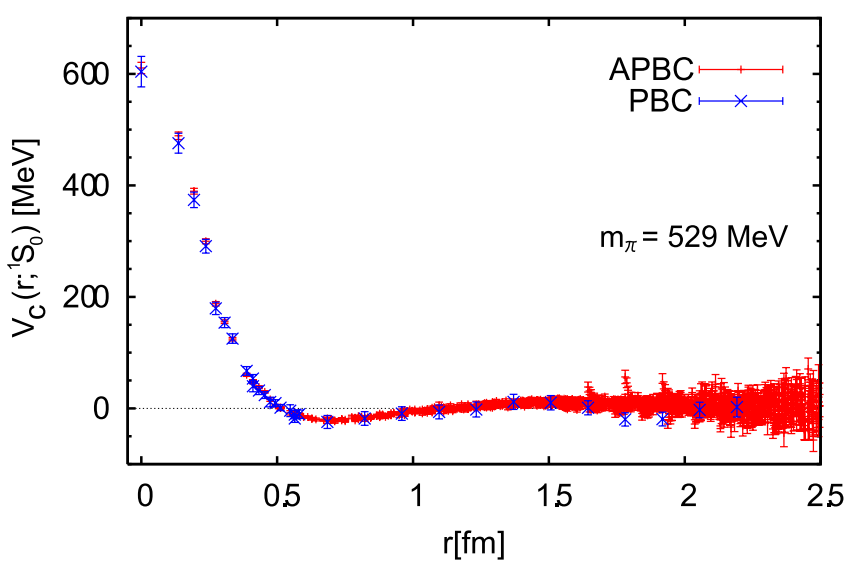

Figure 3: The central $\mathrm{NN}$ potentials for the ${ }^{1} \mathrm{~S}_{0}$ state with $\mathrm{APBC}$ (red bars) and PBC (blue crosses) in quenched QCD at $t / a=9$.

together with the one with $\mathrm{PBC}(E \simeq 0)$. Fluctuations of the data with APBC at large distances $(r>1.5 \mathrm{fm})$ are mainly caused by contaminations from excited states, together with statistical noises. The potential at $r<1.5 \mathrm{fm}$, on the other hand, is less affected by such contamination. As seen from Fig. 3, the NN potentials are almost identical between $E \simeq 0$ and $E \simeq 50 \mathrm{MeV}$. Namely, the non-locality of the potential with our choice of the interpolating operator is small and the LO potentials shown in the present paper can be used in the energy region at least up to $E \sim 50 \mathrm{MeV}$ without significant modifications.

\section{Numerical result in $(2+1)$-flavor QCD}

To compare our results with empirical data, a key role is played by a full QCD calculation on a large volume with a smaller quark mass. The PACS-CS collaboration is generating $(2+1)$-flavor gauge configurations by employing the Iwasaki gauge action at $\beta=1.90$ on $32^{3} \times 64$ lattice and the $O(a)$-improved Wilson quark (clover) action with a non-perturbatively improved coefficient $c_{\mathrm{SW}}=1.715$ [18]. The lattice scale is determined by $m_{\pi}, m_{K}$ and $m_{\Omega}$, which leads to $a \simeq 0.091$ $\mathrm{fm}$. Thus, the spatial extension amounts to $L \simeq 2.90 \mathrm{fm}$. The periodic boundary condition is imposed along the spatial direction, while the Dirichlet boundary condition on the time-slice $t=32$ is imposed along the temporal direction. The wall source on the time-slice is located at $t=0$ with the Coulomb gauge fixing. Fig. 4 shows the full QCD results of the central force for $m_{\pi} \simeq 702$ $\mathrm{MeV}: V_{\mathrm{C}}\left(r ;{ }^{1} S_{0}\right)$ and $V_{\mathrm{C}}^{\mathrm{eff}}\left(r ;{ }^{3} S_{1}\right)$ are obtained from BS wave functions on the time-slices $t=8$ and $t=9$, respectively, where the ground state saturations are achieved within error bars. Similar to the quenched results, a repulsive core surrounded by an attractive well can be seen in full QCD.

\section{Concluding remarks}

We have discussed the basic notion of the nucleon-nucleon potential and its field-theoretical derivation from the equal-time Bethe-Salpeter amplitude in QCD. By construction, the non-local potential defined through the projection of the wave function to the interaction region (the inner 


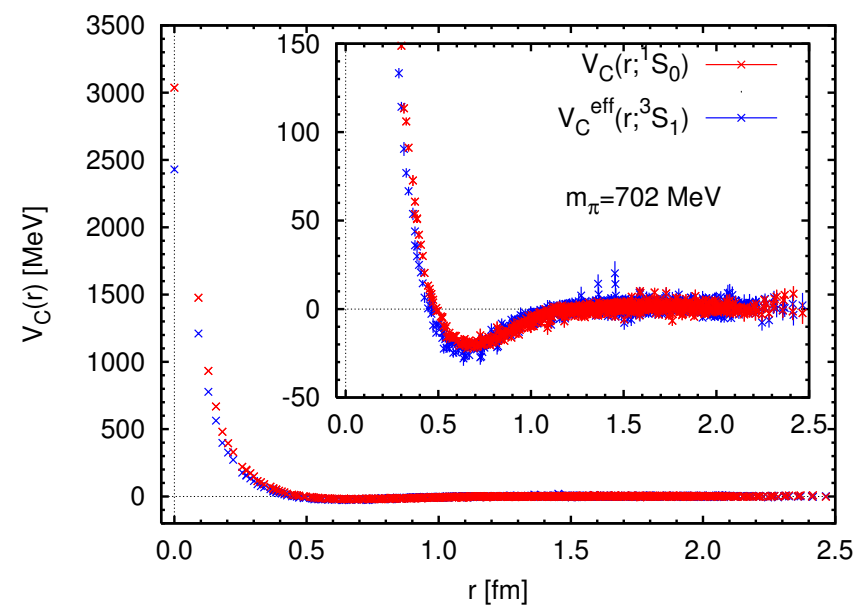

Figure 4: Central and effective central potentials in (2+1)-flavor lattice QCD simulations with $m_{\pi}=702$ $\mathrm{MeV}$.

region) correctly reproduces the asymptotic form of the wave function in the region beyond the range of the nuclear force (the outer region). Thus the observables such as the phase shifts and the binding energies can be calculated after extrapolating the potential to the infinite volume limit. Non-locality of the potential can be taken into account successively by making its velocity expansion, which introduces the velocity-dependent local potentials. The leading order terms of such velocity expansion are the central potential and the tensor potential, and the next-to-leading order term is the spin-orbit potential.

As an exploratory study, we carried out quenched lattice QCD simulations of the two-nucleon system in a spatial box of the size $(4.4 \mathrm{fm})^{3}$ with the pion mass $m_{\pi}=380,529,731 \mathrm{MeV}$. The $\mathrm{NN}$ potential calculated on the lattice at low energy is found to have all the characteristic features expected from the empirical NN potentials obtained from the experimental NN phase shifts, namely the repulsive core surrounded by the attractive well for the central potential. As for the tensor potential obtained by the coupled channel treatment of the ${ }^{3} S_{1}$-state and the ${ }^{3} \mathrm{D}_{1}$-state, appreciable attraction at long and medium distances is found. Phenomenological fit of the tensor potential strongly suggests the existence of the one-pion-exchange in its long range part.

There are a number of directions to be investigated on the basis of our approach:

1. Determination of the velocity dependence is important in deriving the NN potentials useful for the wide range of scattering energies. As mentioned in $\operatorname{Sec} 5.2$, studies along this line using the anti-periodic boundary condition in the spatial direction have been already started [17].

2. To derive the realistic NN potentials on the lattice, it is necessary to carry out full QCD simulations with dynamical quarks. As mentioned in Sec 6, studies along this line with the use of the (2+1)-flavor QCD configurations generated by PACS-CS Collaboration are currently under way [16].

3. The hyperon-nucleon (YN) and hyperon-hyperon (YY) potentials are essential for understanding the properties of hyper nuclei and the hyperonic matter inside the neutron stars. 
Recently, studies of the YN potential in quenched QCD [19] and in full QCD [20] have been started.

4. The three-nucleon force is thought to play important roles in nuclear structures and in the equation of state of high density matter [21]. Since the experimental information is scarce, simulations of the three nucleons on the lattice may lead to the first principle determination of the three-nucleon potential in the near future.

\section{References}

[1] R. Machleidt and I. Slaus, J. Phys. G27, R69 (2001).

E. Epelbaum, H. W. Hammer and U. G. Meissner, arXiv:0811.1338 [nucl-th].

[2] H. Yukawa, Proc. Math. Phys. Soc. Japan, 17, 48 (1935).

[3] R. Jastrow, Phys. Rev. 81, 165 (1951).

[4] H. Heiselberg and V. Pandharipande, Ann. Rev. Nucl. Part. Sci. 50, 481 (2000).

[5] M. Oka, K. Shimizu and K. Yazaki, Prog. Theor. Phys. Suppl. 137, 1 (2000). Y. Fujiwara, Y. Suzuki and C. Nakamoto, Prog. Part. Nucl. Phys. 58, 439 (2007).

[6] Y. Nambu, Phys. Rev. 106, 1366 (1957).

[7] M. Lüscher, Nucl. Phys. B 354, 531 (1991).

[8] M. Fukugita, Y. Kuramashi, M. Okawa, H. Mino and A. Ukawa, Phys. Rev. D 52, 3003 (1995). S. R. Beane, P. F. Bedaque, K. Orginos and M. J. Savage, Phys. Rev. Lett. 97, 012001 (2006).

[9] N. Ishii, S. Aoki and T. Hatsuda, Phys. Rev. Lett. 99, 022001 (2007).

[10] S. Aoki, T. Hatsuda and N. Ishii, Comput. Sci. Disc. 1, 015009 (2008) [arXiv:0805.2462 [hep-ph]].

[11] Y. Kuramashi, Prog. Theor. Phys. Suppl. 122, 153 (1996) [arXiv:hep-lat/9510025].

[12] K. Nishijima, Phys. Rev. 111, 995 (1958). W. Zimmermann, Nuov. Cim. 10, 597 (1958). R. Haag, Phys. Rev. 112, 669 (1958).

[13] S. Okubo and R.E. Marshak, Ann. Phys. (NY) 4, 166 (1958).

[14] R. Tamagaki and W. Watari, Prog. Theor. Phys. Suppl. 39, 23 (1967).

[15] S. Aoki et al. (CP-PACS Coll.), Phys. Rev. D71, 094504 (2005).

[16] N. Ishii, S. Aoki and T. Hatsuda, PoS LAT2008 (2008) 155 [arXiv:0903.5497 [hep-lat]].

[17] S. Aoki, J. Balog, T. Hatsuda, N. Ishii, K. Murano, H. Nemura and P. Weisz, PoS LAT2008 (2008) 152 [ arXiv:0812.0673 [hep-lat] ].

[18] Y. Kuramashi, PoS LAT2008 (2008) 018 [ arXiv:0811.2630 [hep-lat] ].

[19] H. Nemura, N. Ishii, S. Aoki and T. Hatsuda, Phys. Lett. B 673, 136 (2009).

[20] H. Nemura, N. Ishii, S. Aoki and T. Hatsuda [PACS-CS Collaboration], PoS LAT2008 (2008) 156 [ arXiv:0902.1251 [hep-lat] ].

[21] S. C. Pieper, V. R. Pandharipande, R. B. Wiringa and J. Carlson, Phys. Rev. C 64, 014001 (2001). 